\title{
Similarity of ideal gas flow at different scales
}

\author{
WANG Moran (王沫然) \& LI Zhixin (李志信)
}

Department of Engineering Mechanics, Tsinghua University, Beijing 100084, China

Correspondence should be addressed to Wang Moran (email: moralwang99@mails.tsinghua.edu.cn)

Received March 7, 2003

\begin{abstract}
The similarity of ideal gas flow at different scales is investigated analytically and numerically. With the compressible and rarefied effects considered, two dimensionless parameters, Mach number and Knudsen number, are proposed as the similarity criterions, because the Reynolds number can be expressed by the Mach number and the Knudsen number of ideal gases. A DSMC method is used to simulate flows at different scales with the same Ma and $K n$, including subsonic channel flows and the supersonic flows over a hot plate. Comparisons between the results of different scales show that the normalized fields of macroscopic quantities are the same. This confirms the similarity. Especially, the results indicate that the micro flow are similar to the rarefied flow of ideal gas, which suggests that many transformations are available from the existing rarefied flow results to the micro flow.
\end{abstract}

Keywords: similarity, ideal gas flow, direct simulation Monte Carlo.

DOI: 10.1360/02ye0072

When the molecular mean free path becomes comparable with an important characteristic length of the flow system, the continuum assumption breaks down and the particle nature of fluid must be explicitly taken into account ${ }^{[1]}$. Such flows, generally named rarefied gas flows, have been investigated widely and deeply due to their important applications in aeronautics and astronautics. The rarefied gas flow can be described by the Boltzmann equation from a molecular point of view. However, it is rather difficult to obtain analytical and numerical solutions of the Boltzmann equation. The direct simulation Monte Carlo (DSMC) method proposed by Bird ${ }^{[2]}$ offers a valuable tool for predicting the high $K n$ flow behavior. The DSMC method has been proved and widely applied to the analysis of rarefied gas flow problems in the past several decades ${ }^{[3-9]}$.

In recent years, MEMS/NEMS have been developed and the micro flow has become a hot topic $^{[10]}$. In micro/nano gas flow, the Knudsen number can be quite high even though the gas is of

Nomenclature $a$, sound speed; $P$, pressure; $c_{\mathrm{m}}$, most probable molecular speed; Re, Reynolds number; $d$, molecular diameter; $T$, overall temperature; $H$, height of the computed domain; $T_{\mathrm{g}}$, gas temperature on wall surfaces; $k$, Boltamann constant; $K n$, Knudsen number; $T_{\text {tr }}$, translational temperature; $K n_{\text {local }}$, local Knudsen number; $T_{\text {rot }}$, rotational temperature; $L$, characteristic length; length of the computed domain; $T_{\infty}$, freestream temperature; $m$, molecular mass; $T_{\mathrm{w}}$, wall temperature of cylinder; $M a$, Mach number; $\bar{v}_{\mathrm{m}}$, molecular mean velocity; $n$, number density; $v$, molecular velocity; $\gamma$, specific heat rate; $\mu$, dynamic viscosity; $\lambda$, mean free path; $v$, kinetic viscosity; $\rho$, density; $\varepsilon_{\text {rot }}$, rotational energy; $\zeta$, number of internal degree of freedom; $\sigma_{\mathrm{T}}$, total collision cross-section; $\Delta t$, time step; $\varepsilon_{\mathrm{r}}$,translational energy; $\Delta x$, size of a cell; $\infty$, freestream; tr, translational mole; w, wall surfaces; rot, rotational mode. 
high density due to the small characteristic length of the micro system. The classical N-S equations are not able to describe the flow accurately ${ }^{[11]}$, so the DSMC method has been tried and used to predict micro gas flow and heat transfer since the middle of the $1990 \mathrm{~s}^{[1,12-18]}$.

Since the rarefied gas flows on an original size scale have been investigated maturely and the understanding of micro/nano flow mechanism may have a significant bearing on the development and design of MEMS/NEMS, the current paper is an attempt to discuss the similarity of ideal gas flow at different scales. Firstly, the similarity is theoretically analyzed. Then, the DSMC method is developed to simulate gas flows and heat transfer at different scales to verify the similarity numerically.

\section{Similarity analysis}

If the traditional incompressible flows in two different-size systems are considered similar, the dimensionless parameter, Reynolds number, must be equal. For a compressible flow, a more dimensionless parameter, Mach number, plays an important role as well, while in the rarefied or micro gas flow, the Knudsen number must be also considered since it affects the velocity and temperature boundary conditions directly. However, there is a relationship between the three parameters, and one can be expressed in terms of the other two.

The Reynolds number is the ratio of inertial forces to viscous ones

$$
R e=\frac{U L}{v},
$$

where $v$ is the gas kinetic viscosity.

The Mach number is the ratio of flow velocity to the speed of sound

$$
M a=\frac{U}{a},
$$

where $a$ is the sound speed in gas.

The Mach number is a dynamic measure of fluid compressibility and may be considered as the ratio of inertial forces to elastic ones.

According to the molecular theory of gas, for the ideal gas made of smooth rigid elastic spherical molecules with a repelling force from each other, the kinematic viscosity is related to the mean free path $^{[19]}$

$$
v=\frac{\mu}{\rho}=0.499 \lambda \bar{v}_{\mathrm{m}} \approx \frac{1}{2} \lambda \bar{v}_{\mathrm{m}}
$$

where $\mu$ is the gas dynamic viscosity, $\lambda$ the molecular mean free path and $\bar{v}_{\mathrm{m}}$ the mean molecular speed, somewhat higher than the sound speed $a$,

$$
\bar{v}_{\mathrm{m}}=\sqrt{\frac{8}{\pi \gamma}} a,
$$

where $\gamma$ is the gas specific heat rate. 
Combining eqs. (1)-(4), the required relation can be obtained

$$
K n=\sqrt{\frac{\pi \gamma}{2}} \frac{M a}{R e} .
$$

Eq. (5) shows that as long as two of the three dimensionless numbers are specific, the third is also determinate. Then, arbitrary two of the three numbers can be proposed as the similarity criterions. In the present paper the Mach number and Knudsen number are selected as the similarity criterions for ideal gas flow in different-scale systems. That is to say, when the pressure is not too high $(P<1000 \mathrm{~atm})$, the temperature is not too high or too low $(30 \mathrm{~K}<T<2500 \mathrm{~K})$, the Mach number is not too high $(M a<5)$ and there is no reaction, the gas can be treated as an ideal gas and the gas flows at different scales are similar ${ }^{[20]}$. The similarity is confirmed by numerical simulations of the inner and external gas flows at different scales using the developed DSMC method.

\section{Numerical method}

\subsection{DSMC procedure}

DSMC is a molecular-based statistical simulation method for rarefied gases flow introduced by $\operatorname{Bird}^{[2]}$. It is a numerical solution method to solve the dynamic equations for real gases flow by at least thousands of simulated molecules. Each simulated molecular represents a large number of real molecules. Under the assumption of molecular chaos and gas rarefaction, the binary collisions are only considered. Therefore, the molecular motion and their collisions are uncoupling if the computational time step is shorter than the physical collision time. The molecules motion, interactions with boundaries and mutual interactions are calculated where momentum and energy conservation are kept. The macroscopic flow characteristic is obtained statistically by sampling molecular properties in each cell. At the beginning of the procedure, simulated particles are equally statistically distributed in the cells. Within one time step, all particles are first advanced according to their individual velocity and interact with the boundaries, and then they are indexed to be recognized. In each cell, a certain number of collision pairs are selected by no-time-counter (NTC) method and collisions are calculated. Repeat these steps until the statistical errors are small enough. The DSMC method can be used to simulate non-equilibrium and unsteady gas flow. The steady flow field is obtained with a sufficiently long simulated time.

\subsection{Model and time step}

The variable hard sphere (VHS) model ${ }^{[2]}$ incorporates the hard-sphere scattering law for collision, and treats the cross-section of the molecules as a function of the relative translational energy during the collision. Therefore, it is adopted to determine real diameter of gas molecule $(z)$ and the total collision cross-section $\left(\sigma_{\mathrm{T}}\right)$.

The time step used in DSMC method, $\Delta t$, should be less than the mean collision time, so that the particle movement and their collision may be uncoupled, i.e.

$$
\Delta t<\Delta x / c_{m}^{\prime},
$$


where $c_{m}^{\prime}$ is most probable molecular speed of the gas. The cell size $\Delta x$ is taken as less than half of the mean free path in the current simulated situation, i.e.

$$
K n_{\text {local }}=\lambda / \Delta x \geqslant 2 \text {. }
$$

By the traditional computational fluid dynamics (CFD), this constraint may be expressed as a Courant-Friedrichs-Lewy (CFL) number. So

$$
\mathrm{CFL}=\frac{c_{\mathrm{m}}^{\prime} \Delta t}{\Delta x}<1 .
$$

Physically, this condition requires the particle to reside in the same cell for at least a few time steps behind it to interact with other particles. This ensures that its information can be distributed properly throughout the domain of computation. Therefore, for DSMC, the CFL number is not stability constraint, but rather a physical requirement. Violation of this condition may yield solutions that are not physically realistic. In the simulations to be presented, the value of CFL is set at about $0.2^{[12]}$.

\subsection{Boundary condition}

The temperature of the walls is set equal. When the simulated particle collides with the wall, the diffuse reflection model is used to determine the reflection. In this model, the emission of the impinging molecules is not correlated with the pre-impingement state of the molecules. The outgoing velocity is randomly assigned according to a half-range Maxwellian distribution determined by the wall temperature. This is also known as the full thermal and momentum accommodation.

\subsection{Macroscopic physical quantities}

The macroscopic physical quantities of the flow field are obtained by statistic calculation as follows:

$$
\rho=n m,
$$

where $n$ is gas molecule number density and $m$ the molecular mass.

$$
T=\left(3 T_{\text {tr }}+\zeta T_{\text {rot }}\right) /(3+\zeta),
$$

where $T_{\text {tr }}$ is the translational temperature, $T_{\text {rot }}$ is the rotational temperature and $\zeta$ is the number of internal degrees of freedom. The vibrational energy is neglected. There are

$$
\begin{aligned}
\frac{3}{2} k T_{\mathrm{tr}} & =\overline{m v^{2}}-\bar{m} \cdot \bar{v}^{2}, \\
T_{\mathrm{rot}} & =\frac{2}{k}\left(\overline{\varepsilon_{\mathrm{rot}}} / \zeta\right),
\end{aligned}
$$

where $k$ is the Boltzmann constant, $\varepsilon_{\text {rot }}$ is the molecular rotational energy and the overbar, $\overline{()}$, represents sample average.

According to the state equation of ideal gas, the pressure of the gas is

$$
P=\rho R T=n k T \text {. }
$$




\section{Code verifications}

The developed DSMC code is verified by two typical flow cases-Couette flow and Poiseuille flow. The simulated results are compared with the analytical slip theory based on the continuum assumption $^{[21]}$.

In the Couette flow, the upper plate moves at $U=300 \mathrm{~m} / \mathrm{s}$, and the temperature of the plates is $273 \mathrm{~K}$. The gas flows initially at the normal temperature and pressure (NTP). The comparisons of dimensionless velocity and temperature distributions between the DSMC simulations and the analytical results are shown in fig. 1 , where $T_{\mathrm{g}}$ is the gas temperature on the wall surfaces and $T_{\mathrm{w}}$ is the wall temperature. The present DSMC simulation gives macroscopic characteristic distributions that agree very well with the analytical results. The relative scattering error is less than 2.5 percent.
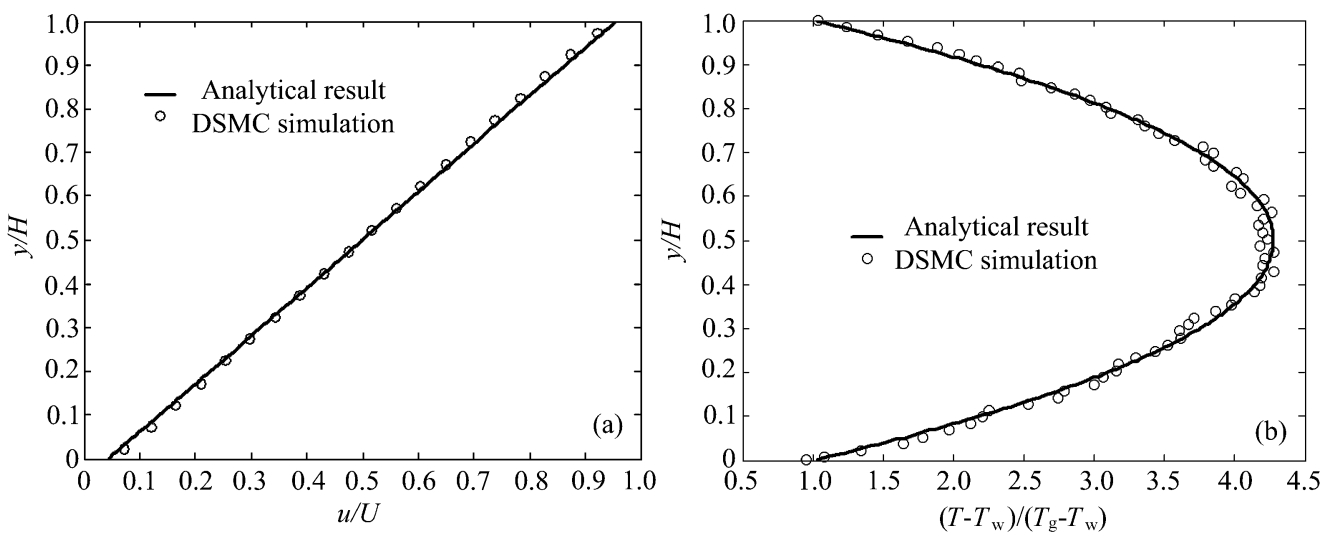

Fig. 1. Comparisons between the DSMC simulation and the analytical results. (a) Dimensionless velocity distribution; (b) dimensionless temperature distribution.

In the Poiseuille flows, two cases are simulated, whose inlet-outlet pressure boundaries are $0.6-0.15$ and $0.2-0.04$ atm, respectively. Both the wall and the coming nitrogen gas temperatures are $300 \mathrm{~K}$. The implicit pressure boundary condition is implemented in the light of refs. [22, 23]. The dimensionless velocity profiles near the outlet normalized by the centerline velocities are compared with the first-order and high-order slip approximations of continuum theory ${ }^{[21]}$ (fig. 2). The local Knudsen numbers are labeled in the figures. In the small Knudsen number case, the DSMC results agree well with both the first-order and the second-order analytical calculations. And in the large Knudsen number case, the DSMC method and the second-order approximation agree well; however, the first-order profile moves away from the DSMC results, with the highest difference occurring at the channel wall.

The good agreement between the DSMC results and the analytical approximations verifies the developed code. 

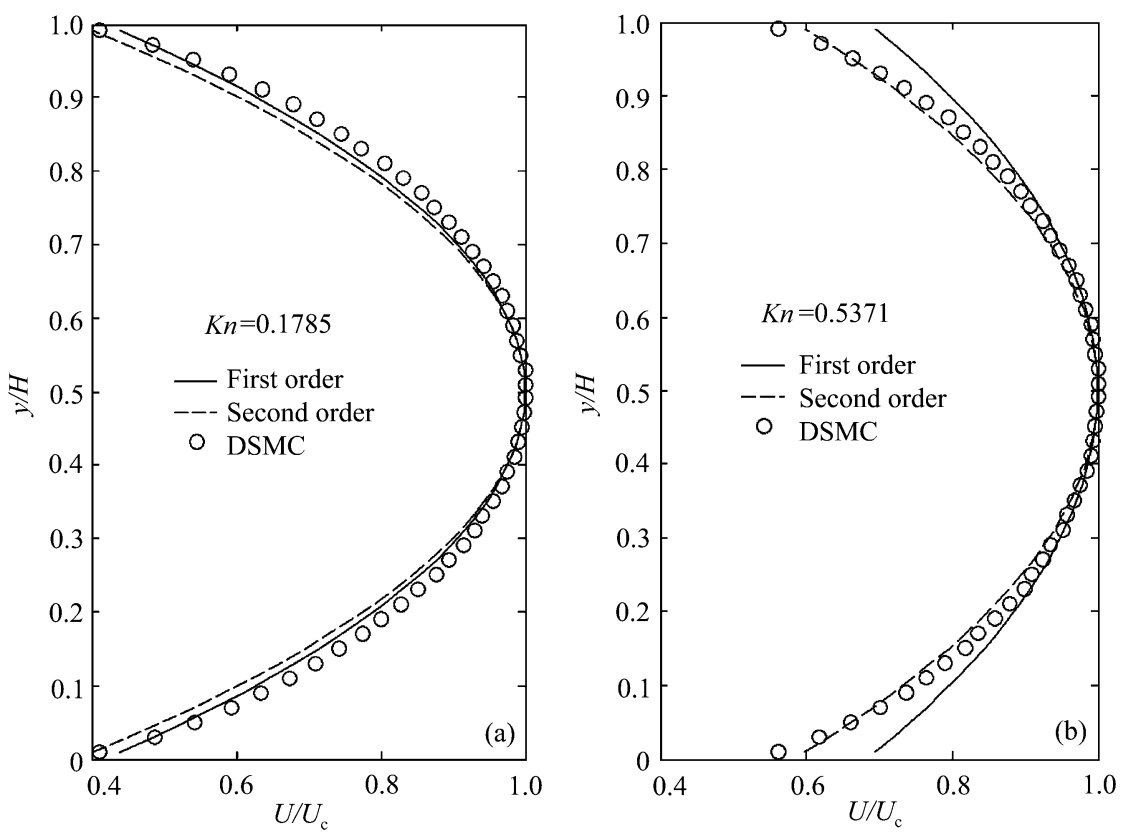

Fig. 2. Comparison of velocity profile of micro Poiseuille flow at different Knudsen numbers.

\section{Results and discussions}

Both the subsonic inner flow and the supersonic external flow are simulated using the developed DSMC code. The results are compared between different scales.

In the subsonic inner flow simulation, the aspect ratio of the channels is set at 5 for all cases. The rectangular cells $(100 \times 60)$ and four subcells $(2 \times 2)$ in a cell are used. The working fluid is nitrogen, whose parameters are shown in table 1 . The studied cases are listed in table $2 . H$ is the height of the channel, which ranges from meter to tens of nanometer. The freestream temperature $T_{\infty}$, as well as the wall temperature $T_{\mathrm{w}}$, is set at $300 \mathrm{~K}$. The Knudsen number and Mach number of the coming gas flow are unit and 0.57 , respectively for all cases. The total number of calculated molecules exceeds $10^{5}$ and the total sample is over $10^{6}$ times in each case.

Table 1 Parameters of $N_{2}$

\begin{tabular}{ccccc}
\hline$m / \mathrm{kg}$ & $\zeta$ & $d_{\mathrm{ref}} / \mathrm{m}$ & $T_{\text {ref }} / \mathrm{K}$ & $\omega$ \\
\hline $4.65 \mathrm{E}-26$ & 2 & $4.17 \mathrm{E}-10$ & 273 & 0.74 \\
\hline
\end{tabular}

Table 2 Simulation cases of subsonic flow in channels

\begin{tabular}{ccccccccc}
\hline Case & $H / \mathrm{m}$ & $K n$ & $M a$ & $T_{\infty} / \mathrm{K}$ & $T_{\mathrm{w}} / \mathrm{K}$ & $n_{\infty}$ & $N_{\text {tol }}$ & $N_{\text {sample }}$ \\
\hline 1 & $1 \mathrm{e} 0$ & & & & & $1.294384 \mathrm{E}+18$ & 101133 & 1011050 \\
2 & $1 \mathrm{e}-3$ & \multirow{2}{*}{0.57} & 300 & 300 & $1.294384 \mathrm{E}+21$ & 101253 & 1005050 \\
3 & $1 \mathrm{e}-6$ & & & & & $1.294384 \mathrm{E}+24$ & 101552 & 1010050 \\
4 & $1 \mathrm{e}-8$ & & & & & $1.294384 \mathrm{E}+26$ & 101329 & 1010050 \\
\hline
\end{tabular}

The $x$-direction velocity and temperature profiles at the inlet and the outlet at different scales 
are shown in fig. 3. Both macro-scale rarefied gas and micro-scale gas have the same slip velocity and jump temperature as long as the Knudsen numbers are equal. The velocity and temperature profiles are almost the same at different scales. The fluctuations come from the statistical error. Dimensionless density and pressure distributions along the midline of the channels normalized by the freestream quantities at different scales are shown in fig. 4 . Though the densities and the pressure drops on micro scales are much larger than those on macro scales, the normalized ones by the freestream quantities become consistent. The figures show that the inner flows at different scales are similar and have the same $K n$ and $M a$.
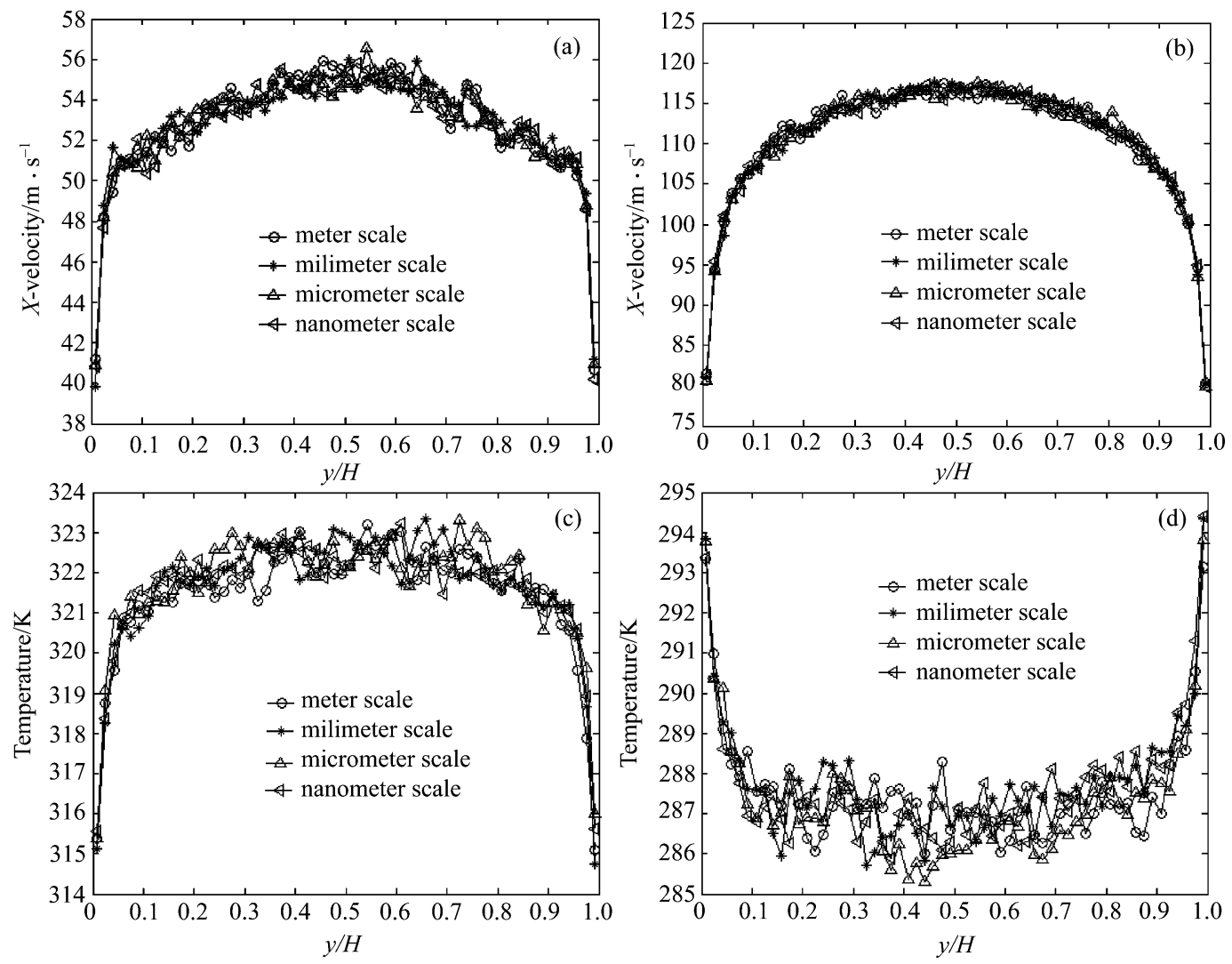

Fig. 3. Velocity and temperature profile at inlet and outlet at different scales. (a) Velocity profiles at the inlet; (b) velocity profiles at the outlet; (c) temperature profiles at the inlet; (d) temperature profiles at the outlet.

The supersonic and external cases are also investigated. The supersonic flows over a hot plate at different scales are simulated using the developed DSMC code. The physical model is shown in fig. 5. The flow field is $L \times H$, and the plate, $L_{\mathrm{w}}$ in length, lies at the bottom of the field. In the simulation, the aspect ratio is set at $1: 0.6$, and the length of the plate is set at $L_{\mathrm{w}}=0.9 \mathrm{~L}$. Other parameters of simulated cases are listed in table 3. 

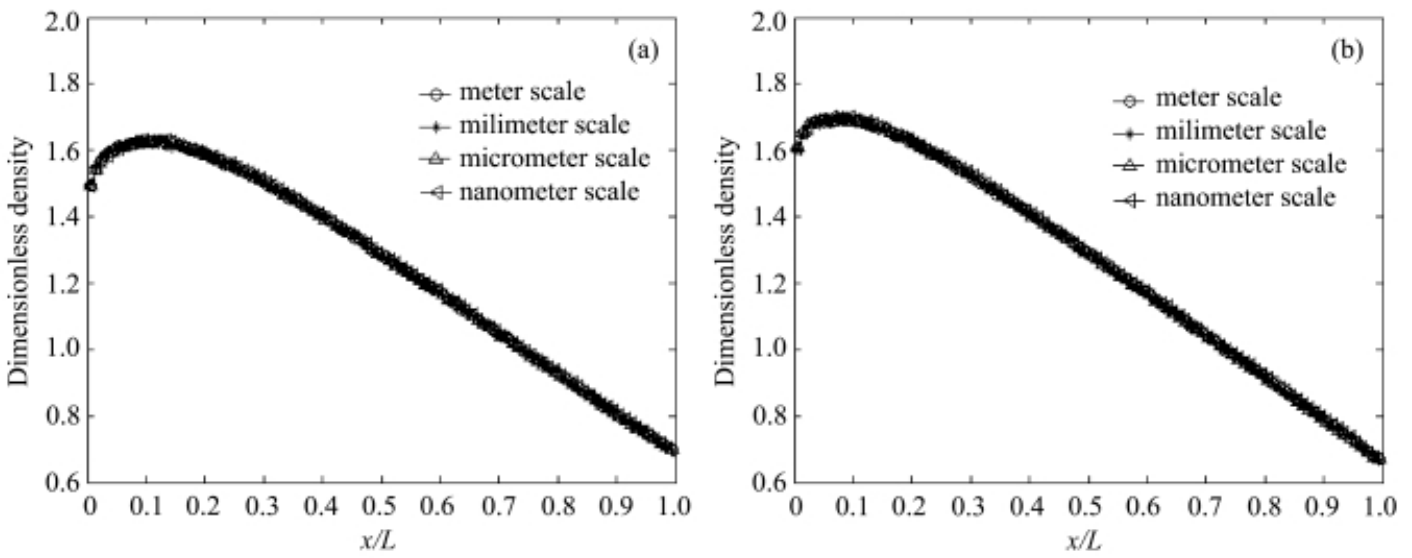

Fig. 4. Dimensionless macroscopic characteristic distributions along the midline of the channels normalized by the freestream densities at different scales. (a) Density distributions; (b) pressure distributions.

Table 3 Simulation cases of supersonic flow over a plate

\begin{tabular}{ccccccccc}
\hline Case & $L / \mathrm{m}$ & $K n$ & $M a$ & $T_{\infty} / \mathrm{K}$ & $T_{\mathrm{w}} / \mathrm{K}$ & $n_{\infty}$ & $N_{\text {tol }}$ & $N_{\text {sample }}$ \\
\hline 1 & $1 \mathrm{e} 0$ & & & & & $1 . \mathrm{E}+20$ & 37640 & 500000 \\
2 & $1 \mathrm{e}-3$ & & & & & $1 . \mathrm{E}+23$ & 37693 & 500000 \\
3 & $1 \mathrm{e}-6$ & 0.0216 & 4.0 & 300 & 500 & $1 . \mathrm{E}+26$ & 37453 & 500000 \\
4 & $1 \mathrm{e}-8$ & & & & & $1 . \mathrm{E}+28$ & 37660 & 500000 \\
\hline
\end{tabular}

The results are shown in fig. 6 . The $x$-velocity

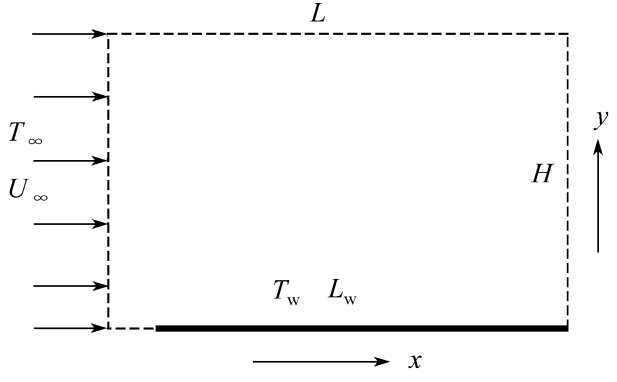

Fig. 5. Physical model of the supersonic flow over plate. and temperature profiles at the end of the plate at different scales are plotted in fig. 6(a) and (b). Fig. 6 (c) shows the dimensionless density distributions at the end of the plate and fig. 6(d) shows the dimensionless pressure distributions along the bottom of the flow domain. The velocity and temperature distributions are the same for different scales. The density and pressure distributions differ at different scales, but the dimensionless distributions normalized by the freestream quantities are the same. The relative error is very small due to the supersonic gas velocity, so the distributions at different scales almost fall on the same curve.

\section{Concluding remarks}

The similarity of ideal gas flow at different scales has been investigated. The Mach number and the Knudsen number are proposed as the similarity criterions for ideal gas flow according to the relationship between $R e, M a$ and $K n$. The DSMC method is developed, with which the similarity is confirmed by the simulations of subsonic inner flows and supersonic external flows. Comparisons between the results at different scales show that with the same freestream Mach 

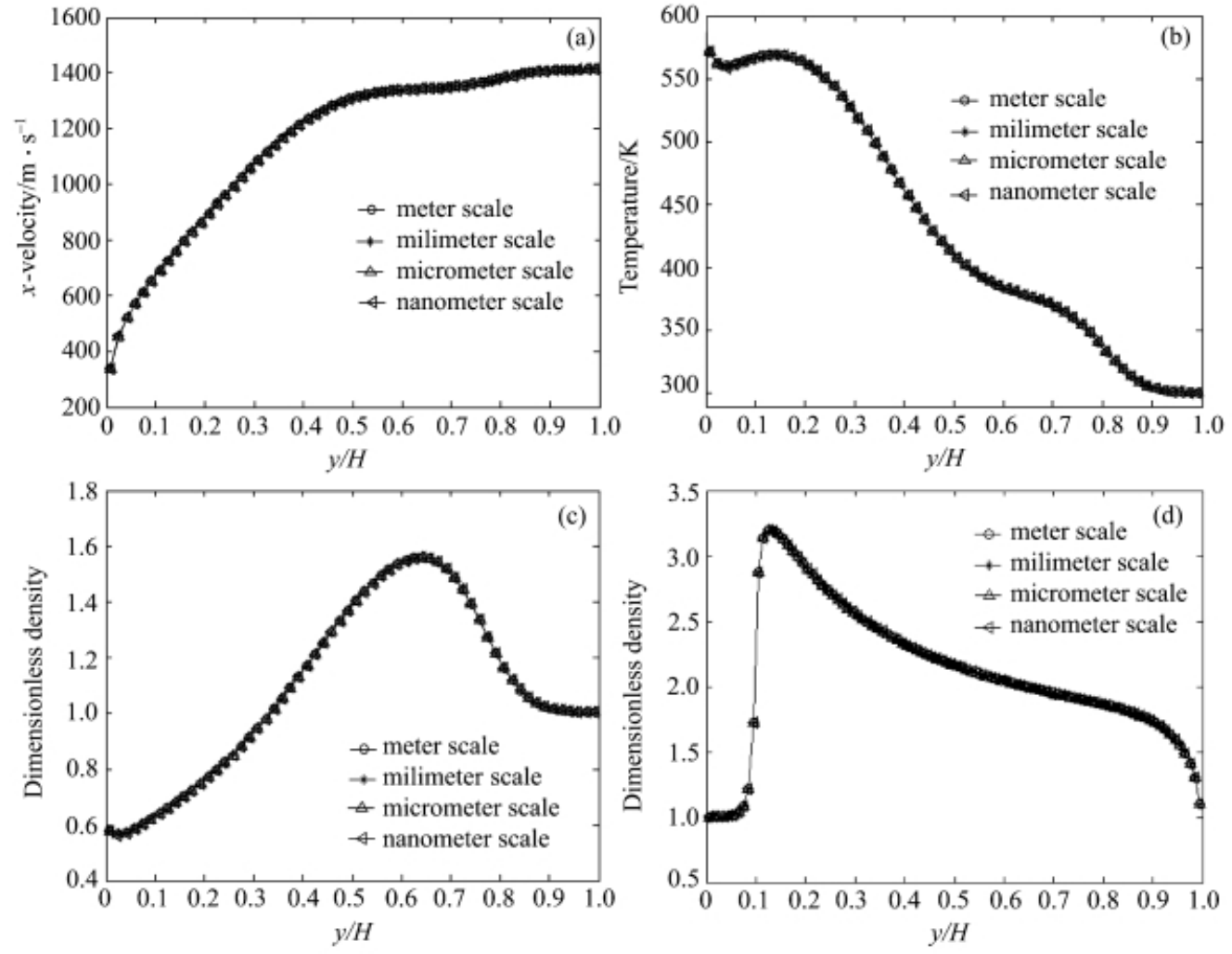

Fig. 6. Simulation results of supersonic flow over a hot plate. (a) $x$-velocity profile at the end of the plate; (b) temperature profile at the end of the plate; (c) dimensionless densities profile at the end of the plate normalized by freestream densities; (d) dimensionless pressure along the bottom line.

number and Knudsen number the velocity and the temperature fields are the same at different scales. Though the density and the pressure fields are quite different in magnitude, the dimensionless ones normalized by the freestream quantities are also the same. This shows that they are similar by the Mach number and Knudsen number.

Especially, the similarity between micro and rarefied ideal gas flow by $M a$ and $K n$ suggests that many existing results of rarefied flows can be transferred to the micro flows under the same boundary conditions.

Acknowledgements The authors would like to thank Shen Ching at Institute of Mechanics, the Chinese Academy of Sciences and M. Macrossan at the Department of Mechanical Engineering, University of Queensland. This work was supported by the National Natural Science Foundation of China (Grant No. 59995550-2) and the National Key Basic Research and Development Program of China (1999033106).

\section{References}

1. Karniadakis, G. E., Beskok, A., Micro Flows: Fundamentals and Simulation, New York: Springer-Verlag, 2002.

2. Bird, G. A., Molecular Gas Dynamics and the Direct Simulation of Gas Flows, Oxford: Clarendon Press, 1994.

3. Oran, E. S., Oh, C. K., Cybyk, B. Z., Direct simulation Monte Carlo: Recent advances and applications, Annual Review of Fluid Mechanics, 1998, 30: 403-441. 
4. Koura, K., Improved null-collision technique in the direct simulation Monte Carlo method: Application to vibrational relaxation of nitrogen, Computers Math. Applic., 1998, 35: 139-154.

5. Cheng, C. H., Liao, F. L., DSMC analysis of rarefied gas flow over a rectangular cylinder at all Knudsen numbers, J. Fluid Engineering of ASME, 2000, 122: 720-729.

6. Pan, L. S., Ng, T.Y., Xu, D. et al., Determination of temperature jump coefficient using the direct simulation Monte Carlo Method, J. Micromechanics and Microengineering, 2002, 12: 41-52.

7. Pan, L. S., Liu, G. R., Lam, K.Y., Determination of slip coefficient for rarefied gas flow using direct simulation Monte Carlo, J. Micromechanics and Microengineering, 1999, 9: 89-96.

8. Kannenberg, K. C., Boyd, I. D., Three-dimensional Monte Carlo simulations of plume impingement, J. Thermophysics and Heat Transfer, 1999, 13: 226-235.

9. Yan, F., Farouk, B., Discontinuous wall temperature distribution induced gas flow in an enclosure at high Knudsen number, in Symposiums of International Conference of Heat and Mass Transfer, France, 2002.

10. Ho, C. M., Tai, Y. C., Micro-electro-mechanical-systems(MEMS) and fluid flows, Annu. Rev. Fluid Mech., 1998, $30: 579$ -612 .

11. Mohamed Gad-el-Hak, The fluid mechanics of microdevices — The freeman scholar lecture, Journal of Fluids Engineering, 1999, 121:5-33.

12. Liou, W. W., Fang, Y., Heat transfer in microchannel devices using DSMC, J. MEMS, 2001, 274-279.

13. Wu, J. S., Tseng, K. C., Analysis of micro-scale gas flow with pressure boundaries using direct simulation Monte Carlo Method, J. Computers and Fluids, 2001, 30: 711—735.

14. Hadjiconstantinou, N. G., Simek, O., Constant-wall-temperature Nusselt number in micro and nano-channels, J. Heat Transfer of ASME, 2002, 124: 356-364.

15. Alexeenko, A. A., Levin, D. A., Gimelshein, S. F. et al., Numerical simulation of high-temperature gas flow in a millimeter-scale thruster, J. Thermophysics and Heat Transfer, 2002, 16: 10-16.

16. Xue, H., Fan, Q., Shu, C., Prediction of micro-channel flows using direct simulation Monte Carlo, Probabilistic Engineering Mechanics, 2000, 15: 213-219.

17. Sun, Q., Boyd, I. D., Numerical simulation of gas flow over microscale airfoils, J. Thermophysics and Heat Transfer, 2002, 76: $171-179$.

18. Wang, X., Wang, Q.W., Tao, W. Q. et al., Simulation of rarefied gas flow and heat transfer in microchannels, Science in China, Ser. E, 2002, 45(3): 321-327.

19. Chapman, S., Cowling, T. G., The Mathematical Theory of Nonuniform Gases, 3rd ed., Cambridge: CUP, 1970.

20. Shen, C., Raredied Gas Dynamics (in Chinese), Beijing: National Defence Industry Publishing House, 2003.

21. Beskok, A., Simulations and Models for Gas Flows in Microgeomitries, Ph. D. Dissertation, Cambridge: MIT 1996.

22. Nance, R. P., Hash, D. B., Hassan, H. A., Role of boundary conditions in Monte Carlo Simulation of MEMS devices, Journal of Thermalphysics and Heat Transfer, 1998, 12: 447-449.

23. Liou, W. W., Fang, Y. C., Implicit boundary conditions for direct simulation Monte Carlo method in MEMS flow predictions, Computer Modeling in Engineering \& Science, 2000, (4): 119-128. 